\title{
Ultrasound Pixel-based Beamforming with Phase Alignments of Focused Beams
}

\author{
Nghia Q. Nguyen and Richard W. Prager
}

\begin{abstract}
We previously developed unified pixel-based beamforming to generate high-resolution sonograms, based on field pattern analysis. In this framework, we found the transmit wave-shape away from the focus could be characterized by two spherical pulses. These correspond to the maximal and minimal distances from the imaging point to the active aperture. The beamformer uses this model to select the highest-energy signals from backscattered data. A spatiotemporal interpolation formula is used to provide a smooth transition in regions near the focal depth where there is no dominant reflected pulse. In this paper, we show that the unified pixel-based approach is less robust at lower center frequencies. The interpolated data is suboptimal for a longer transmit wave-shape. As a result, the spatial resolution at the focal depth is lower than that in other regions. By further exploring the field pattern, we propose a beamformer that is more robust to variations in beam-width. The new method, named coherent pixel-based beamforming, aligns and compounds the pulse data directly in the transition regions. In simulation and phantom studies, the coherent pixel-based approach is shown to outperform unified pixel-based in spatial resolution. It helps regain optimal resolution at the focal depth while still maintaining good image quality in other regions. We also demonstrate the new method on in vivo data where its improvements over unified pixel-based are demonstrated on scanned objects with a more complicated structure.
\end{abstract}

Index Terms - ultrasound, pixel-based beamforming, virtual source, coherent beamforming, image quality, spatial resolution.

\section{INTRODUCTION}

Pixel-based (PB) beamforming has been developed recently as a way to generate B-mode images with improved resolution. It avoids interpolation artefacts by performing dynamic focusing individually for every point in the image [1], [2]. Similar to synthetic aperture (SA) imaging [3], [4], a high-resolution image can be formed by compounding the low-resolution images created from individual pulse-echo sequences. Unlike SA imaging that involves the use of defocused pulses, however, PB beamforming is based on a conventional focussed ultrasonic transmission. Because the beam is narrower, data superposition is usually limited to a small number of transmitreceive cycles. In its simplest form, PB beamforming generates data at each imaging point using only signals backscattered from the nearest transmit event [1], [5].

Attempts to extend the superposition to include transmission events further across the active aperture lead to the use of the virtual source element (VSE) technique [6]. In this approach, a source is assumed to exist at the transmit focus and a spherical wave is considered to propagate back and forth from this point [7], [8]. In [9], Zemp and Insana showed that the VSE approach can achieve spatial resolution in the nearand far-field similar to that at the focal depth. The spherical wave-shape, however, is only a good approximation within a limited angle. Outside this angle, the wave shape assumption is invalid. The VSE approach therefore requires that we discard these data, which could otherwise benefit the image reconstruction [6], [10]. The lack of a way to incorporate these data may create errors in the B-mode image especially when there is high echogenicity around the focal depth. Such errors can be avoided by setting the focal length outside the imaging region [11]. This strategy, however, broadens the transmit beam and compromises the spatial resolution.

In an earlier study [12], we developed a unified PB beamformer that is implemented with a highly focused transmit beam. Our motivation was to demonstrate high-resolution reconstruction at all image depths using a conventional focussed transmit-receive acquisition sequence. Through field pattern analysis, we modeled the transmitted wave-shape at points away from the focus as two weak pulses. The arrival times of these pulses correspond to the maximal and minimal distances from the imaged point to the active aperture. This leads to a generalization of the VSE technique that is valid over the entire imaging region. Based on this finding, we developed an algorithm to compute the appropriate time delay for each received signal in the beamformer. It combines the VSE technique to extract data associated with the highest energy received pulse and an heuristic interpolation formula to give a smooth transition in regions where the VSE technique is not applicable. The unified PB beamformer was shown to generate high-resolution images when applied to both experimental and in vivo data.

In a companion paper [13], however, we found that the unified PB was less robust when applied to data acquired with a lower center frequency. As the transmit beam becomes broader, the unified PB requires more data from the heuristic interpolation formula to generate a smooth transition around the focal depth. This degrades the image resolution at the focal depth because the heuristic formula is suboptimal for selecting focussed signals in the transition between the two pulses. This is in contrast to the performance of many conventional algorithms where the image has best resolution at the focal depth. It motivates us to investigate the field pattern further, update the unified PB beamformer, and develop a new method that is more robust to variations in the center frequency.

The paper is organized as follows. In Section II, we briefly summarize the field pattern profile and the unified PB beamformer developed in [12]. We then present a new method, named coherent pixel-based beamforming based on 


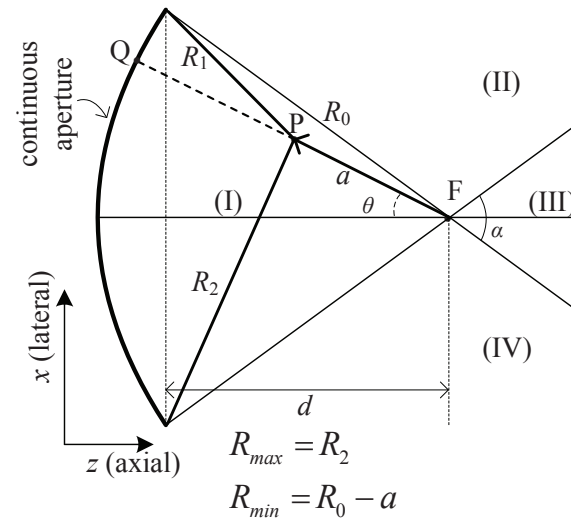

(a)

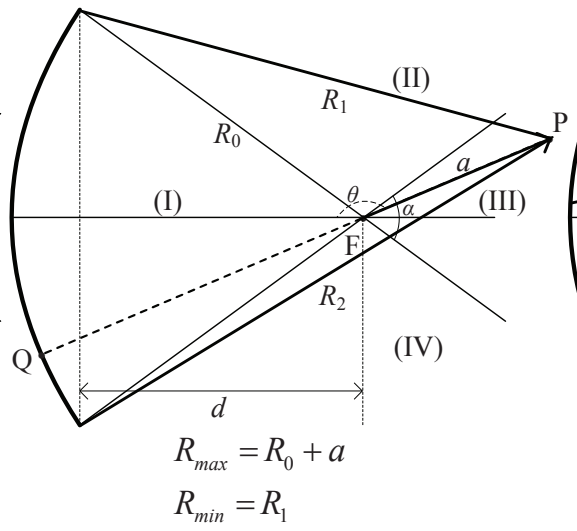

(b)

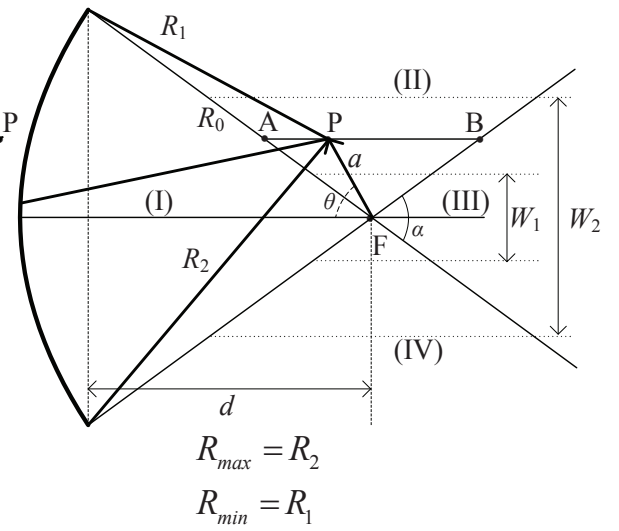

(c)

Fig. 1. Geometries for field profile analysis and time delay calculations with a transmit beam. The transmit delays associated with the elements of the active linear aperture are represented by the curvature of the aperture in the axial-lateral plane (from [12], Fig. 1). The same notation is used in all figures (a)-(c).

an extension of this analysis. In Section III, we demonstrate the new method and compare it to the unified PB beamformer using simulations [14], [15] and experimental data acquired with an instrumented ultrasound machine [16]. Beamformer performance is evaluated qualitatively and quantitatively based on the generated images. We also demonstrate the coherent PB beamformer on in vivo data from an ultrasound scan of the carotid artery. The imaging results verify the robustness of the new method to structural complexity of the scanned object in clinical applications. Finally, we summarise and draw some conclusions in Section IV.

\section{Methods}

\section{A. Pixel-based Beamforming}

Similarly to SA imaging [4], PB beamforming uses timedelay information to align and assemble signals received at individual elements for each image point $\mathrm{P}$. The beamformed signal is generated by

$$
g\left(\mathbf{x}_{p}\right)=\sum_{i}^{N_{t}} \sum_{j}^{N_{r}} w_{i, j} r_{i, j}\left(\tau_{p}(i, j)\right),
$$

where $N_{t}$ is the number of transmits, $N_{r}$ is the number of receiving elements in the active aperture, $r_{i, j}(t)$ is the waveform or echo RF trace received on element $j$ with transmit $i$, the $w_{i, j}$ 's are apodization coefficients, and $\tau_{p}(i, j)$ is the time delay used to extract signals from the received waveform $r_{i, j}(t)$.

The receive time-delay (between back-scatter and reception at the probe) is calculated straightforwardly based on the distance between $\mathrm{P}$ and the corresponding receiving element (given the sound-speed $c$ is known). Thus, beamformer performance depends on appropriate calculation of the transmit time delays from the probe excitation to the intended target $\mathrm{P}$. Conventionally, this delay is calculated by assuming the transmit pulse is spherical and propagates from the center of the active aperture [2], [3]. This assumption, however, only holds within a limited angular extent for each transmit [4]. Far from the transmit centerline, the conventional timedelay results in adding noise and off-target interference to the beamformed image, rather than the echo from the intended target. This limits the image from being generated with a large number of transmits.

In this study, we have analyzed the pressure field to develop a better time delay calculation. The analysis is based on a combination of the impulse response method [17] and simulation using the Field II program [14], [15]. In the following section we briefly summarize our earlier work that lays the foundations for the unified pixel-based beamforming in [12]. We then further extend it to develop the new pixel-based beamformer that is the focus of the present paper.

\section{B. Extended Field Pattern Analysis}

We take the transmit delay at individual elements into account and model the aperture as a continuous uniform arc with a focal depth $d$ and radius $R_{0}$ [9]. From the focal point $\mathrm{F}$, we divide the imaging plane into four regions using the limited angle $\alpha$ in the virtual source approach [7], [8]. The regions, denoted from (I) to (IV) clockwise, are shown in Figs. 1(a)(c) with different scenarios for the image point $\mathrm{P}$ with respect to the aperture. A point in region (IV) will have the same treatment as one in region (II), thus, it is not shown in the figure.

In the analysis, we use four geometrical distances to characterize the transmit wave-shape. These are $R_{\max }$ and $R_{\min }$ which are the maximal and minimal distances from $\mathrm{P}$ to the aperture, and $R_{1}$ and $R_{2}$ from $\mathrm{P}$ to the edges of the aperture. For $\mathrm{P}$ in region (I) (Fig. 1(a)), the pressure field is given by

$$
\begin{aligned}
p\left(\mathbf{x}_{p}, t\right) & =\frac{\rho c \beta_{q}}{\pi} v\left(t-\frac{R_{\text {min }}}{c}\right) \\
& -\frac{\rho c}{2 \pi}\left[\beta_{1} v\left(t-\frac{R_{1}}{c}\right)+\beta_{2} v\left(t-\frac{R_{2}}{c}\right)\right]
\end{aligned}
$$

where $v(t)$ is the excitation, $\rho$ is the equilibrium density of the medium, and $\beta_{1}, \beta_{2}$, and $\beta_{q}$ are angular coefficients associated to $R_{1}, R_{2}$, and $R_{\text {min }}$ (derived from point $\mathrm{Q}$ on the aperture). Depending on the position of $\mathrm{P}$ inside region $(\mathrm{I})$, the maximal distance $R_{\max }$ is either $R_{1}$ or $R_{2}$. The wave-shape has three 


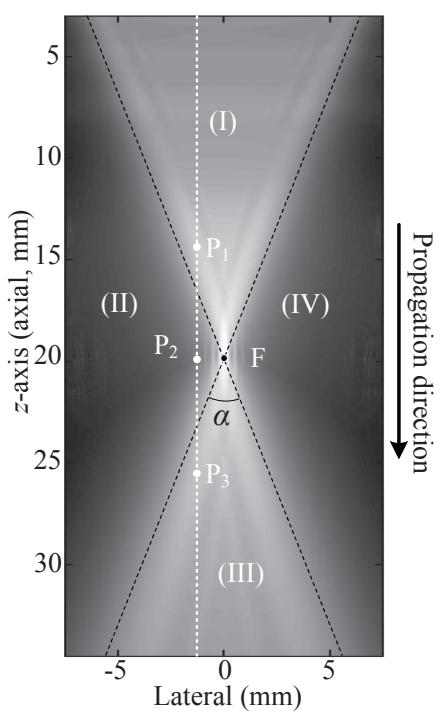

(a)

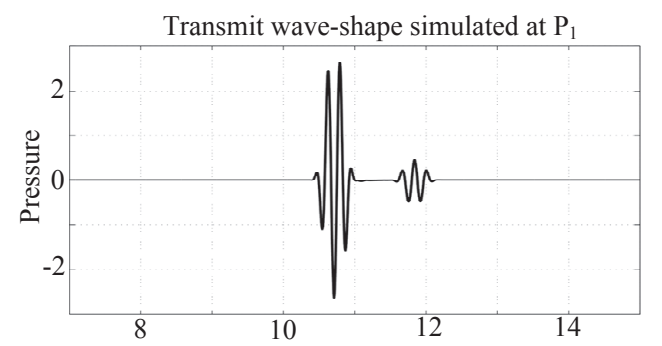

Transmit wave-shape simulated at $\mathrm{P}_{3}$

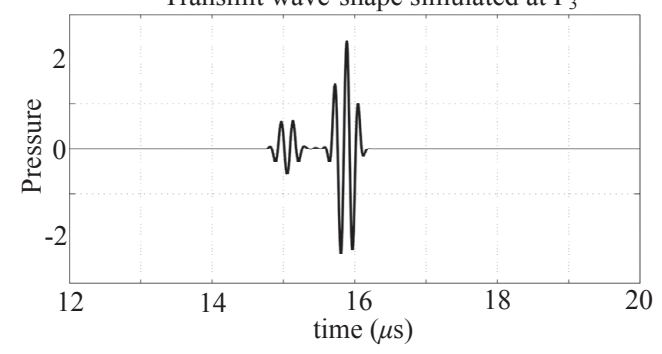

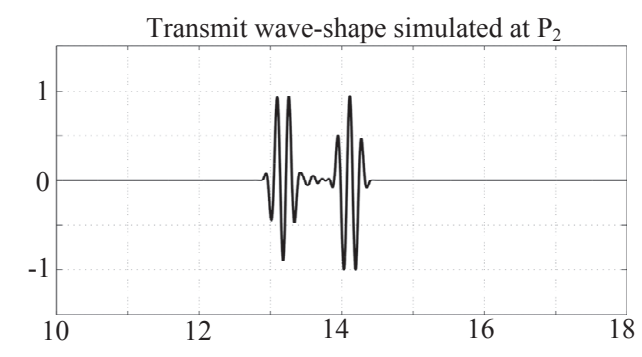

Transmit wave-shape simulated at $\mathrm{F}$

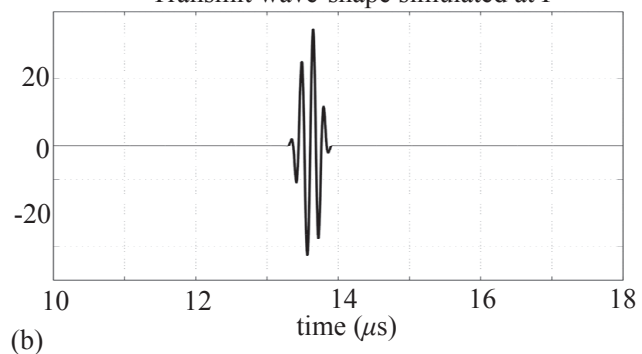

Fig. 2. (a) The pattern field from one transmit event simulated after the ULA-OP system and transducer LA523 by using Field II program. (b) Transmit wave-shapes generated at imaging points $\mathrm{P}_{1}, \mathrm{P}_{2}, \mathrm{P}_{3}$, and the focus $\mathrm{F}$. All wave-shapes are normalized to the amplitude of that simulated at $\mathrm{P}_{2}$.

pulses, however, it is dominated by the highest energy pulse associated with $R_{\min }$.

For $\mathrm{P}$ in region (III) (Fig. 1(b)), we have the pressure field

$$
\begin{aligned}
p\left(\mathbf{x}_{p}, t\right) & =\frac{\rho c}{2 \pi}\left[\beta_{1} v\left(t-\frac{R_{1}}{c}\right)+\beta_{2} v\left(t-\frac{R_{2}}{c}\right)\right] \\
& -\frac{\rho c \beta_{q}}{\pi} v\left(t-\frac{R_{\max }}{c}\right),
\end{aligned}
$$

where $\beta_{q}$ is the angular coefficient associated with $R_{\max }$. In this region, the transmit wave-shape has three pulses but is dominated by the one associated with $R_{\max }$. Also depending on the position of $\mathrm{P}$, the pulse with either $R_{1}$ or $R_{2}$ corresponds to the minimal distance $R_{\text {min }}$.

For $\mathrm{P}$ in region (II) (Fig. 1(c)),

$$
p\left(\mathbf{x}_{p}, t\right)=\frac{\rho c}{2 \pi}\left[\beta_{1} v\left(t-\frac{R_{1}}{c}\right)-\beta_{2} v\left(t-\frac{R_{2}}{c}\right)\right] .
$$

These two pulses are associated with $R_{1}$ and $R_{2}$. In this case $R_{1}$ and $R_{2}$ are actually $R_{\min }$ and $R_{\max }$ respectively. The pressure field contains only two pulses and neither of them consistently dominates the wave-shape. The first pulse, associated with $R_{1}$, has a higher magnitude near region (I), while the second pulse, associated with $R_{2}$, becomes larger near region (III).

At the focal point $\mathrm{F}$, all $R_{\max }, R_{\min }, R_{1}$ and $R_{2}$ are the same and the pulses are merged into one highly focused pulse. At other positions, however, the excitation pulses at transmit elements are out-of-phase. The wave-shape is separated into several out-of-focus pulses. Based on the analysis, we approximate the wave-shape as a combination of the two pulses corresponding to the distances $R_{\max }$ and $R_{\min }$ from $\mathrm{P}$ to the active aperture. We denote them as the min pulse and max pulse, and use the information of their arrival times to form the transmit time delay calculation.
This analysis also reveals the relationship between the phases of the max and min (distance) pulses in the transmit wave-shape. For $\mathrm{P}$ in region (I), the min pulse corresponds to $R_{\text {min }}$, while the max is one of those associated to $R_{1}$ or $R_{2}$. From Eq. (2), we can see that in either case, the max pulse is phase inverted with respect to $R_{\text {min }}$. Similarly for $\mathrm{P}$ in region (III) where the wave-shape is generated in Eq. (3), the min pulse corresponds to either $R_{1}$ or $R_{2}$, and in both cases are phase-inverted with respect to the $R_{\max }$ pulse. Furthermore, it is clear from (4) that the two pulses are phase inverted with respect to each other for $\mathrm{P}$ in region (II).

We confirm these new findings relating to phase inversion using Field II simulations. First, we generate the field pattern of the transmit beam, modeled after the ULA-OP system described in Section III, and show it in Fig. 2(a). In Fig. 2(b), we plot the simulated transmit wave-shapes at four imaging points $\mathrm{P}_{1}, \mathrm{P}_{2}, \mathrm{P}_{3}$, and at the transmit focus $\mathrm{F}$. The positions of these points are shown in Fig. 2(a). In these plots, the waveshape at $\mathrm{F}$ includes only one pulse with the highest magnitude. At other points, the pulse is broken into two much weaker pulses, almost completely out-of-phase with each other.

Our analysis has some limitations. Besides the approximation from a discrete linear array to a continuous uniform arc, we ignore the directivity of transducer elements by assuming each of them to be a point source. The analysis also does not include the effects of diffractions near the transducer surface. In the study, however, we are not trying to develop a comprehensive study on the field patterns. Our goal is to extract important information about the transmit beam that can be used to enhance beamformer performance.

\section{Unified Pixel-Based Beamformer [12]}

The unified PB approach generates focusing data with a time delay calculation based on the arrival times of the min and max pulses. As our study focuses on the transmit time 
delay in a pulse-echo sequence, we drop indices $i$ and $j$ in $\tau_{p}(i, j)$ of Eq. (1) and use $\tau_{p}$ to denote the transmit time delay to point $P$.

The selection is obvious in regions (I) or region (III) where the wave-shape is dominated by the min or max pulse respectively. The time delay for $\mathrm{P}$ in region (I) is given by

$$
\tau_{p}=\tau_{p, \min }=\frac{d-a}{c},
$$

and for $\mathrm{P}$ in region (III) it is given by

$$
\tau_{p}=\tau_{p, \max }=\frac{d+a}{c} .
$$

These calculations include the delay $\tau_{0}=\left(R_{0}-d\right) / c$ so that the total delay is given with reference to the time at which the last (or central) element fires [12].

In region (II) or (IV), the selection of the dominant pulse is more difficult because the max and min pulses have similar magnitudes. For an artefact-free reconstruction, it is important to have a smooth travel-time field over the whole imaging region. An abrupt change in this field causes artefacts at the corresponding locations in the image. The smooth transition from $\tau_{p, \min }$ in region (I) to $\tau_{p, \max }$ in region (III), however, happens only at the focus F. Thus in [12], we proposed a method to calculate $\tau_{p}$ for $\mathrm{P}$ in region (II) (and the same for region (IV)) as

$$
\tau_{p}=\frac{\left|\mathbf{x}_{b}-\mathbf{x}_{p}\right|}{\left|\mathbf{x}_{b}-\mathbf{x}_{a}\right|} \tau_{p, 1}+\frac{\left|\mathbf{x}_{a}-\mathbf{x}_{p}\right|}{\left|\mathbf{x}_{a}-\mathbf{x}_{b}\right|} \tau_{p, 2}
$$

where $\mathbf{x}_{a}, \mathbf{x}_{b}, \mathbf{x}_{p}$ are vector positions of points $\mathrm{A}, \mathrm{B}$, and $\mathrm{P}$ (see Fig. 1(c)). We assume the origin of these vectors is at the center of the active aperture. The time delays $\tau_{p, 1}$ and $\tau_{p, 2}$ are given by

$$
\tau_{p, 1}=\frac{R_{1}}{c}-\tau_{0} \text { and } \tau_{p, 2}=\frac{R_{2}}{c}-\tau_{0} .
$$

As $\tau_{p, 1}=\tau_{p, \min }$ when $\mathrm{P}$ is at $\mathrm{A}$ and $\tau_{p, 2}=\tau_{p, \max }$ when $\mathrm{P}$ is at $\mathrm{B}$, this calculation provides a linear transition from $\tau_{p, \min }$ to $\tau_{p, \max }$. The time delay calculation in (5)-(7) is a combination comprising the VSE technique plus a linear transition between the highest energy pulses when $\mathrm{P}$ is outside the limited angle.

The imaging data collected at $\mathrm{P}$ in regions (II) and (IV) are important for generating an artefact-free image. For $\mathrm{P}$ that are deep in those regions and far from the beam centerline, however, the transmit pulses are weak. The $\tau_{p}$ in (7) increasingly refers to an even weaker signal between these two pulses. The selected echo data, therefore, is dominated by backscattered noise and off-target interference, and contributes very little information to the reconstructed image. Thus, we use the apodization coefficients $w_{i, j}$ to regularize the signals and limit the noise added into the beamformer calculation.

Specifically, we set $w_{i, j}$ equal to 1 for $\mathrm{P}$ in regions (I) or (III). For $\mathrm{P}$ in region (II) or (IV), we set $w_{i, j}$ equal to 1 within an interval $W_{1}$ and 0 outside an interval $W_{2}$ (see Fig. 1(c)). Between these areas, $w_{i, j}$ is set by linearly reducing from 1 to 0 . Parameters $W_{1}$ and $W_{2}$ are selected based on adhoc methods, as well as the visualization and comparison of generated images. Through experiments with various center frequencies, we find the unified PB beamformer can produce an image with good quality using $W_{1} \approx \lambda f_{0} / D$ and $W_{2} \approx$ $3 \lambda f_{0} / D$, where $\lambda$ is the wavelength, $f_{0}$ is the focal depth, and $D$ is the width of the transmit aperture. The term $\lambda f_{0} / D$ is the azimuthal resolution of the transmit beam [17].

\section{Coherent Pixel-Based Beamformer}

In an attempt to incorporate more data from regions (II) and (IV), we propose a new method that directly selects signals associated with the two pulses in the transmit wave-shape. First, we modify the imaging equation in (1) to become

$$
g\left(\mathbf{x}_{p}\right)=\sum_{i}^{N_{t}} \sum_{j}^{N_{r}} w_{i, j} y_{p}(i, j),
$$

where $y_{p}(i, j)$ is synthesized from the data in the received waveform $r_{i, j}(t)$. A major difference of the new method from the unified PB beamformer is that $y_{p}(i, j)$ can be integrated from more than one data point in $r_{i, j}(t)$. In particular, it combines the high energy signals associated with the max and min pulses in the transmit wave-shape at individual imaging points. In order to be of value, this combination will need to take into account the phase inversion between these two pulses.

For $\mathrm{P}$ in region (I), we choose $y_{p}(i, j)=r_{i, j}^{\min }$, which is the data point on $r_{i, j}(t)$ associated with $\tau_{p, \min }$. For $\mathrm{P}$ in region (III), we choose $y_{p}(i, j)=-r_{i, j}^{\max }$, which is the data associated with $\tau_{p, \max }$. This is still the same as for the unified PB beamformer except for the inclusion of a ' - ' sign for phase inversion in region (III).

In region (II) (or region (IV)), we calculate $y_{p}(i, j)$ as

$$
y_{p}(i, j)=\frac{\left|\mathbf{x}_{b}-\mathbf{x}_{p}\right|}{\left|\mathbf{x}_{b}-\mathbf{x}_{a}\right|} r_{i, j}^{1}-\frac{\left|\mathbf{x}_{a}-\mathbf{x}_{p}\right|}{\left|\mathbf{x}_{a}-\mathbf{x}_{b}\right|} r_{i, j}^{2},
$$

where $r_{i, j}^{1}$ and $r_{i, j}^{2}$ are extracted from $r_{i, j}(t)$ with time delays $\tau_{p, 1}$ and $\tau_{p, 2}$, respectively. As $r_{i, j}^{1}=r_{i, j}^{\min }$ for $\mathrm{P}$ at $\mathrm{A}$ and $r_{i, j}^{2}=r_{i, j}^{\max }$ for $\mathrm{P}$ at $\mathrm{B}$, this scheme also provides a smooth transition from the min to the max pulse when $\mathrm{P}$ is outside the limited angle. Notice the '-' sign, again, to account for the phase inversion.

The new method aligns data associated with the max and min pulses propagating to the image point $\mathrm{P}$ from different transmits and combines them, with phase alignment, to generate an artefact-free image. The appropriately delayed and summed waveforms increase the net echo coherence, which improve the lateral resolution of the generated image. We call this approach the coherent pixel-based beamformer.

In this coherent pixel-based approach, we also use a similar apodization scheme based on $W_{1}$ and $W_{2}$ (see Fig. 1(c)) to prevent noise from out-of-focus regions contributing to the image generation process. Because we now have valid data over a greater area, we can set $W_{1}$ and $W_{2}$ wider than those for the unified PB. Specifically, $W_{1} \approx 4 \lambda f_{0} / D$ and $W_{2} \approx 8 \lambda f_{0} / D$, where $\lambda f_{0} / D$ is the azimuthal resolution of the transmit beam. 


\section{RESULTS AND DISCUSSIONS}

\section{A. Data Acquisition and Processing}

In this section, we demonstrate and compare the performance of the coherent PB with other beamformers. They are applied to data acquired with the ULA-OP ultrasound system [16]. The transducer is a linear array of 192 elements (LA523, Esaote SpA, Florence, Italy) with 64 elements active in each transmit event. The transmit beam is focused at $20 \mathrm{~mm}$. In elevation, the aperture is weakly-focused also at $20 \mathrm{~mm}$ using an acoustic lens. The beam is generated with a center frequency $f_{0}$ of $6.0 \mathrm{MHz}$ and a $40 \%$ pulse-echo bandwidth. It is stepped by an element pitch of $0.245 \mathrm{~mm}$ laterally. Received waveforms are sampled at 50 Msamples/s, resulting in an axial sampling interval of $0.0154 \mathrm{~mm}$. In each pulse-echo sequence, the transmit beam is shifted laterally by a step of one element pitch. The dynamic focused image, therefore, is generated with a $0.245 \mathrm{~mm}$ sampling interval in the lateral direction. The PB beamformers improve image quality by generating RF data at points between the scanlines. We choose to generate the PB images with a five-fold finer grid in the lateral direction, which results in a $0.049 \mathrm{~mm}$ sampling interval.

\section{B. Evaluation metrics}

We evaluate each beamformer through the quality of the generated B-mode images, measured with three metrics: spatial resolution, contrast ratio and echo signal-to-noise (eSNR) [18]. The spatial resolution is quantified by measuring the width of simulated point-target responses. In phantom studies, we evaluate the spatial resolution through the speckle size or coherence length of the generated images [19], [20], given by

$$
S_{c}=\int_{-\infty}^{\infty} \frac{C_{X}(\mathbf{x})}{C_{X}(0)} d \mathbf{x}
$$

where $C_{X}(\mathbf{x})$ is the spatial auto-covariance function (ACF) for the RF data, and $d \mathbf{x}$ is the sampling interval 2-D vector. As the PB beamformers only have an impact on the lateral resolution, we measure this metric, denoted by $S_{c x}$, in the lateral direction only.

The eSNR is calculated using the ratio of the ensemble energy of the beamformed echo signals over the noise power. We calculate the echo energy and noise using average/difference over twelve beamformed RF frames acquired in the same scan location. The calculation is performed using kernels that contain no strong reflectors to avoid the effects of sidelobes on the noise power [18].

The contrast ratio between a lesion and the background is quantified by [21]

$$
\mathrm{CR}=\frac{I_{\text {out }}-I_{\text {in }}}{\sqrt{I_{\text {out }}^{2}+I_{\text {in }}^{2}}},
$$

where $I_{\text {in }}$ and $I_{\text {out }}$ are the mean intensities (in decibels) measured inside and outside the lesion, respectively. The term CR has a value of 1 for perfect contrast, and a value of 0 for no contrast between the lesion and background. The background kernel is selected to have the same area as the lesion. To minimise the effect of variations in the attenuation and diffraction of the ultrasound, we choose the kernel as a circular ring enclosing the lesion.
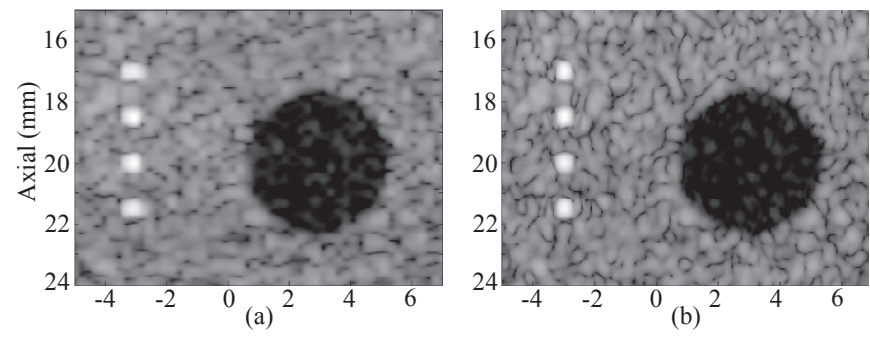

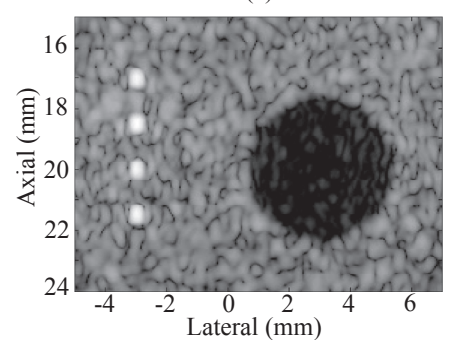

(c)

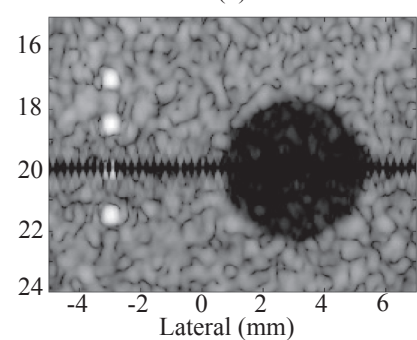

(d)
Fig. 3. Simulated images of a phantom that contains four point-scatterers and an anechoic cyst suspended in a random background. The scatterers are located at $-3 \mathrm{~mm}$ laterally and at $17 \mathrm{~mm}, 18.5 \mathrm{~mm}, 20 \mathrm{~mm}$, and $21.5 \mathrm{~mm}$ in depth, while the cyst is $5 \mathrm{~mm}$ in diameter and centered at $(z, x)=(20,3) \mathrm{mm}$. The images are generated with different strategies: (a) conventional dynamic focusing, (b) unified PB beamformer, (c) coherent PB beamformer, and (d) virtual source element technique where imaging data outside the limited angle is discarded. Images in (b)-(d) are generated with data from 32 transmits. All images are log-compressed and displayed with a dynamic range of $60 \mathrm{~dB}$.

TABLE I

COMPARISON BETWEEN THE UNIFIED PB AND COHERENT PB BEAMFORMERS THROUGH FWHMS OF THE POINT-TARGET RESPONSES.

\begin{tabular}{|c|cccc|}
\hline \hline \multirow{2}{*}{ Beamformer } & \multicolumn{4}{|c|}{ FWHM at a depth of } \\
& $17 \mathrm{~mm}$ & $18.5 \mathrm{~mm}$ & $20 \mathrm{~mm}$ & $21.5 \mathrm{~mm}$ \\
\hline Dynamic focusing & $0.626 \mathrm{~mm}$ & $0.368 \mathrm{~mm}$ & $0.367 \mathrm{~mm}$ & $0.478 \mathrm{~mm}$ \\
Unified PB & $0.305 \mathrm{~mm}$ & $0.308 \mathrm{~mm}$ & $0.346 \mathrm{~mm}$ & $0.318 \mathrm{~mm}$ \\
Coherent PB & $0.299 \mathrm{~mm}$ & $0.292 \mathrm{~mm}$ & $0.263 \mathrm{~mm}$ & $0.280 \mathrm{~mm}$ \\
\hline \hline
\end{tabular}

\section{Simulation}

The simulated data is generated with the Field II program configured to model the ULA-OP system described above. The numerical phantom has a random background with four scatterers suspended at depths from $17 \mathrm{~mm}$ to $21.5 \mathrm{~mm}$ with $1.5 \mathrm{~mm}$ separation. The scatterers line up at $-3 \mathrm{~mm}$ in the lateral direction. There is also an anechoic cyst, $5 \mathrm{~mm}$ in diameter centered at $(z, x)=(20,3) \mathrm{mm}$. The scatterers' strength inside the cyst is 20 times less than those in the background, equivalent to $-26 \mathrm{~dB}$ relative to the root-meansquare amplitude of the background signal. The simulated data contains no noise, thus, we evaluate the images using just the spatial resolution and contrast ratio.

Imaging results generated with different beamformers are shown in Figs. 3. The dynamic focused image, shown in Fig. 3(a), is interpolated to have the same pixel size as the PB beamformed images. Compared to those generated with the unified and coherent PB in Figs. 3(b) and (c), we find the resolution is much improved and blurring artefacts caused by interpolation are removed. To show the advantages of 

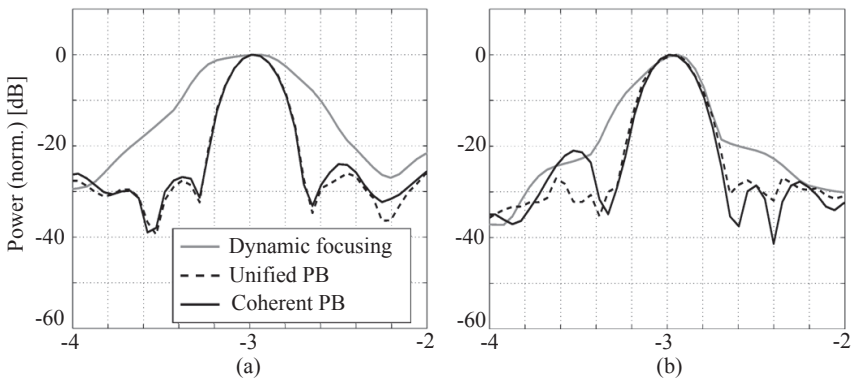

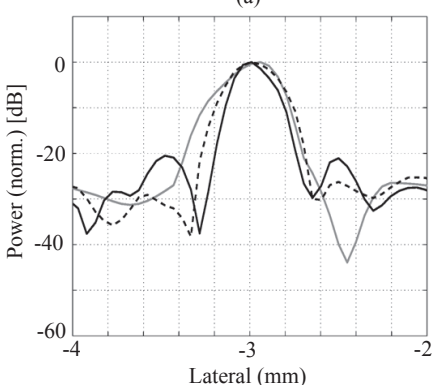

(c)

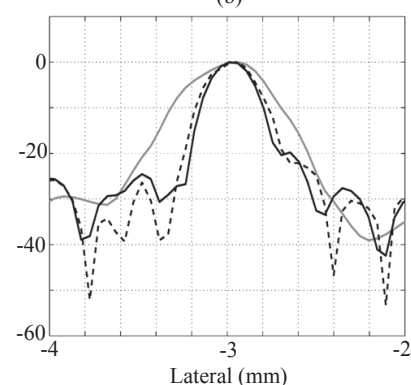

(d)
Fig. 4. Lateral profiles of scatterers at depths of (a) $17 \mathrm{~mm}$, (b) $18.5 \mathrm{~mm}$, (c) $20 \mathrm{~mm}$, and (d) $21.5 \mathrm{~mm}$. The legend in plot (a) also applies to the other plots.

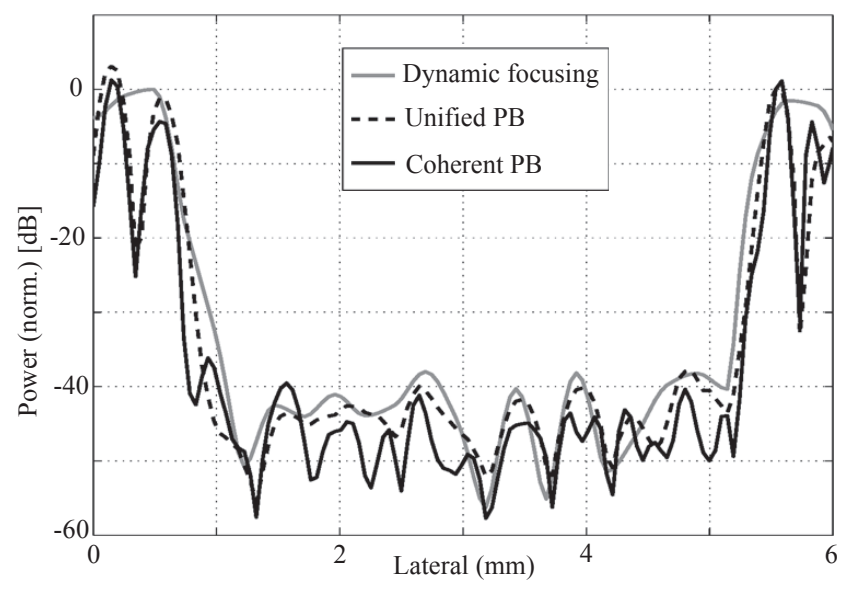

Fig. 5. A lateral section through the anechoic cyst using each of the algorithms at a depth of $20 \mathrm{~mm}$.

integrating data in out-of-focus regions, we apply the VSE calculation to the pre-beamformed signals. In this calculation, we follow suggestions in [6], [10] to discard the data outside the limited angle (regions (II) and (IV) in our analysis). The result is shown in Fig. 3(d) with errors at the focal depth. These errors result from not including all imaging data that is relevant around the focal depth. It is shown in the figure that these errors cannot be corrected using time gain compensation alone.

To enable more detailed comparison, we plot the lateral beamformer profiles of these scatterers in Fig. 4. The responses are not symmetric on these plots because the point scatterers are suspended against a random background. Compared to the unified PB, the coherent PB shows narrower mainlobes at all depths. This indicates the better resolution generated by the coherent PB. The biggest improvement is noticed at $20 \mathrm{~mm}$;
TABLE II

PERFORMANCE METRICS MEASURED ON EXPERIMENTAL DATA OF $6 \mathrm{MHZ}$ CENTER FREQUENCY

\begin{tabular}{cccc}
\hline \hline Beamformers & $\begin{array}{c}S_{c x} \\
\text { (focal, mm) }\end{array}$ & $\begin{array}{c}S_{c x} \\
\text { (far-field, mm) }\end{array}$ & $\begin{array}{c}\text { eSNR } \\
(\mathrm{dB})\end{array}$ \\
\hline Dynamic focusing & $0.53 \pm 0.02$ & $0.74 \pm 0.05$ & $36.39 \pm 3.11$ \\
Unified PB & $0.43 \pm 0.01$ & $0.37 \pm 0.01$ & $39.06 \pm 4.09$ \\
Coherent PB & $0.36 \pm 0.01$ & $0.37 \pm 0.01$ & $38.74 \pm 3.60$ \\
Unified PB & $0.44 \pm 0.02$ & $0.37 \pm 0.01$ & $37.32 \pm 3.41$ \\
(with wider $\left.W_{1,2}\right)$ & & & \\
\hline \hline
\end{tabular}

Azimuthal resolution $\lambda f^{\sharp}=0.332 \mathrm{~mm}$.

TABLE III

PERFORMANCE METRICS MEASURED ON EXPERIMENTAL DATA OF 3.5 MHZ CENTER FREQUENCY

\begin{tabular}{cccc}
\hline \hline Beamformers & $\begin{array}{c}S_{c x} \\
\text { (focal, mm) }\end{array}$ & $\begin{array}{c}S_{c x} \\
\text { (far-field, mm) }\end{array}$ & $\begin{array}{c}\text { eSNR } \\
(\mathrm{dB})\end{array}$ \\
\hline Dynamic focusing & $0.77 \pm 0.02$ & $1.45 \pm 0.05$ & $33.63 \pm 0.51$ \\
Unified PB & $0.74 \pm 0.02$ & $0.70 \pm 0.02$ & $37.09 \pm 0.27$ \\
Coherent PB & $0.62 \pm 0.01$ & $0.70 \pm 0.02$ & $36.55 \pm 0.22$ \\
Unified PB & $0.73 \pm 0.02$ & $0.70 \pm 0.02$ & $34.75 \pm 0.11$ \\
(with wider $\left.W_{1,2}\right)$ & & & \\
\hline \hline
\end{tabular}

Azimuthal resolution $\lambda f^{\sharp}=0.57 \mathrm{~mm}$

this is the depth with the smallest main lobe for coherent PB but is also the worst-performing depth for the unified PB. Thus, the coherent PB is able to recover optimal resolution at the focal depth of the generated images. At this depth, dynamic focusing has a mainlobe width closest to those of other beamformers. This indicates that it achieves its optimal resolution around this region. At other depths, it generates beam profiles with much broader mainlobes. The beamwidths at $-6 \mathrm{~dB}$ are measured and summarized in Table I.

Both unified and coherent PB show improvements over the dynamic focused image in the $\mathrm{CR}$ of the simulated anechoic cyst. For dynamic focusing, $\mathrm{CR}=0.803$, while those measured on the unified and coherent PB images are 0.866 and 0.843 respectively. To enable more detailed comparison, we plot in Fig. 5 a lateral section through the cyst for each image at a depth of $20 \mathrm{~mm}$. In the figure, the coherent PB shows individual scatterer features with a sharper profile indicating the higher spatial resolution. Meanwhile, the dynamic focused image has a smooth curve caused by the blurring interpolation artefacts.

\section{Phantom Study}

Experimental data is acquired by scanning a tissue mimicking phantom (manufactured by the Department of Medical Physics, University of Wisconsin, Madison, WI, USA). The manufacturer reported speeds of sound ranging from $1538 \mathrm{~m} / \mathrm{s}$ to $1551 \mathrm{~m} / \mathrm{s}$ and an attenuation coefficient slope of $0.55 \mathrm{~dB} \mathrm{~cm}^{-1} \mathrm{MHz}^{-1}$.

The first experimental scan includes two hyperechoic circular targets, $5 \mathrm{~mm}$ in diameter, positioned around depths 

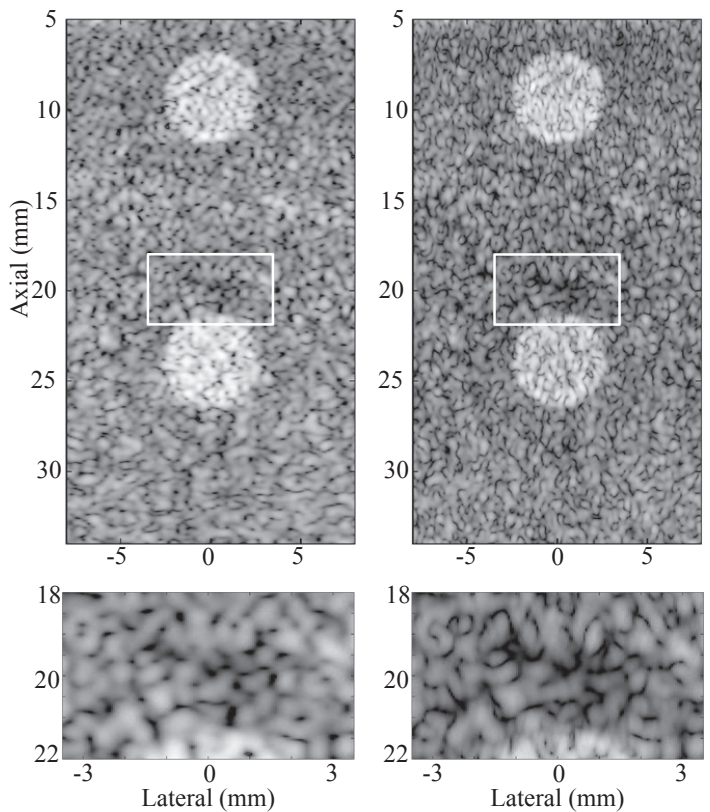

(a)

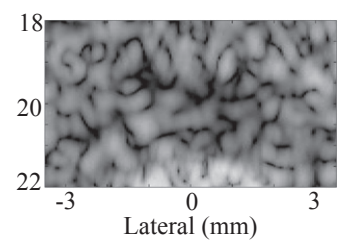

(b)
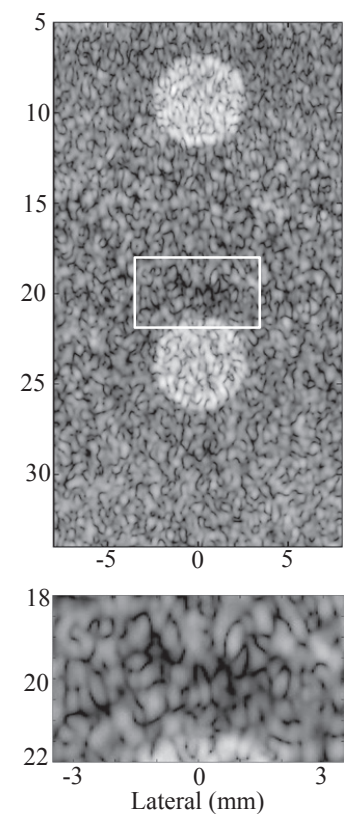

(c)
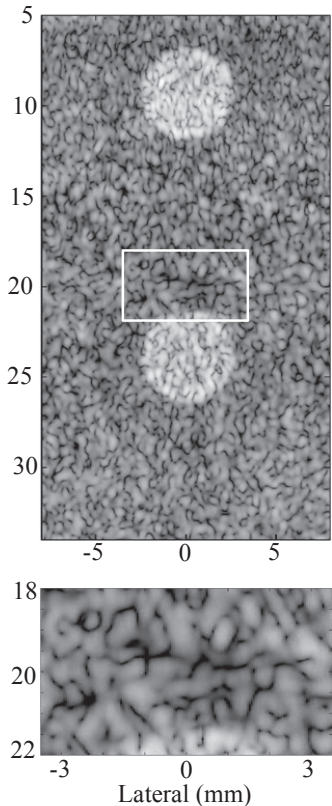

(d)
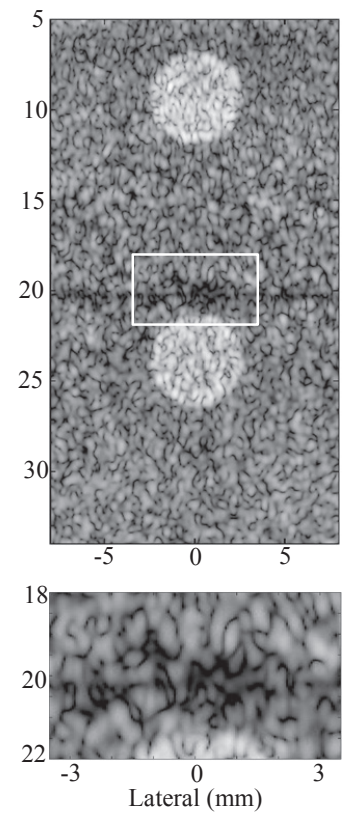

(e)

Fig. 6. (Top) Images of two idealised hyperechoic lesions generated with different beamforming strategies: (a) conventional dynamic focusing, (b) unified PB, (c) coherent PB, (d) unified PB with the same apodization coefficient as the coherent PB, and (e) coherent PB where data is combined without phase inversion. The data is acquired with a center frequency of $6.0 \mathrm{MHz}$. Images (b)-(e) are generated with data from 32 transmits. (Bottom) The regions enclosed by the white rectangles in (a)-(e) are magnified for detailed comparison. All images are log-compressed and displayed with a dynamic range of 45 dB.

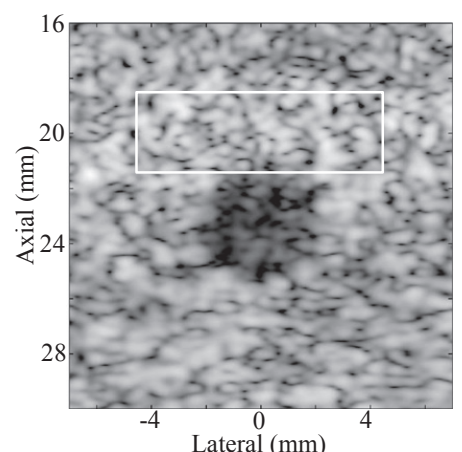

(a)

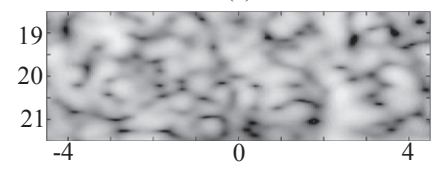

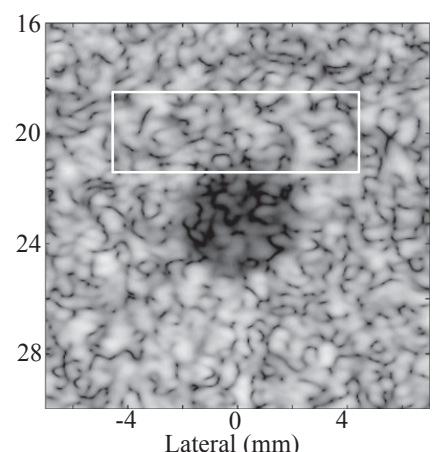

(b)

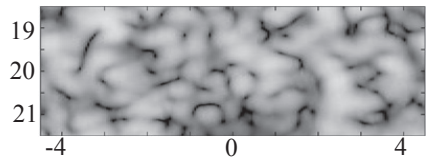

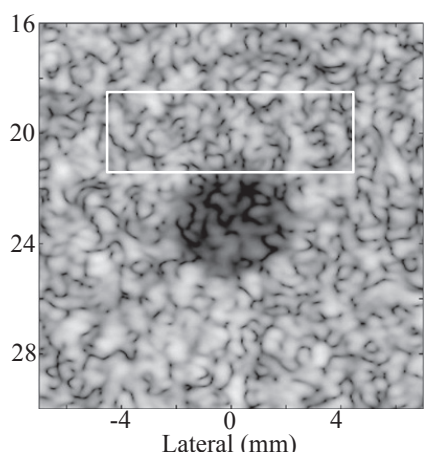

(c)

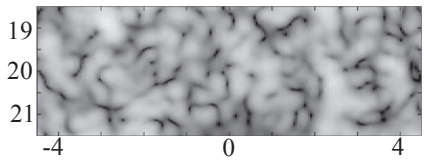

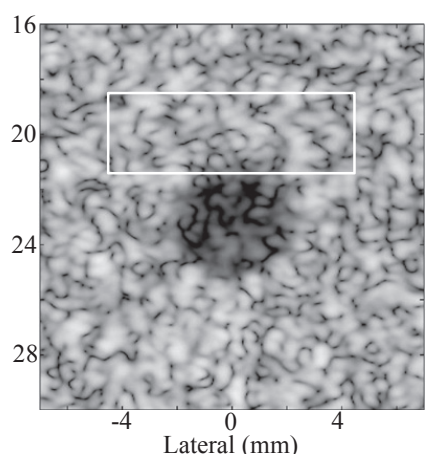

(d)

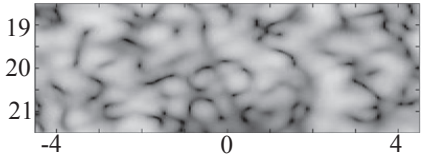

Fig. 7. (Top) Images of an idealised anechoic lesion generated with (a) conventional dynamic focusing, (b) unified PB beamformer, (c) coherent PB beamformer, and (d) unified PB with the same apodization coefficient as the coherent PB. The data is acquired with a center frequency of $3.5 \mathrm{MHz}$. Images (b)-(d) are generated with data from 32 transmits. (Bottom) The regions enclosed by the white rectangles in (a)-(d) are magnified for detailed comparison. All images are log-compressed and displayed with a dynamic range of $40 \mathrm{~dB}$.

of $9 \mathrm{~mm}$ and $23.5 \mathrm{~mm}$. The dynamic focused envelope is interpolated and displayed in Fig. 6(a). The images from the unified and coherent PB beamformers are shown in Figs. 6(b) and (c), respectively. The imaging results show the strengths and limitations of the unified PB beamformer. Although it offers much higher spatial resolution than conventional dynamic focussing, the speckles at the focal depth are wider than those in the far-field. Meanwhile, the coherent PB approach offers the finest speckle pattern at the focal depth. This improvement comes from the data selection associated with the max and min pulses in the out-of-focus regions. This, in turn, allows a larger amount of high-energy data to be combined in the beamforming summation. In the near-field and far-field regions, most of the data are extracted using either the min or max pulse alone, in both coherent and unified PB beamfomers. Hence, the speckle patterns in these regions look the same on both images.

To demonstrate the advantages of the coherent PB more clearly, we generated another unified PB image using the same apodization applied to the coherent PB. Hence the windows 


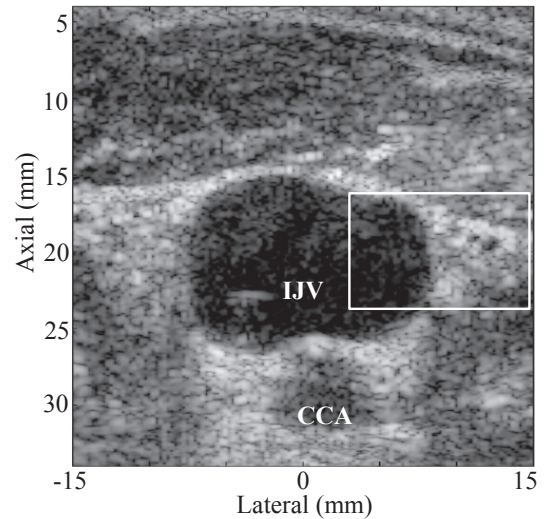

(a)

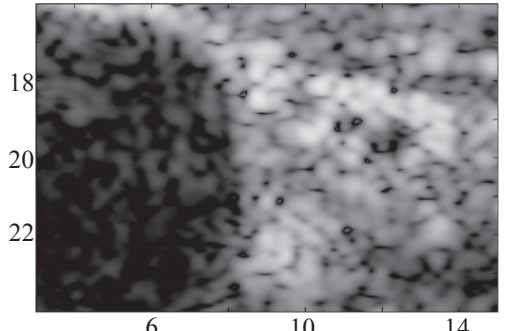

14

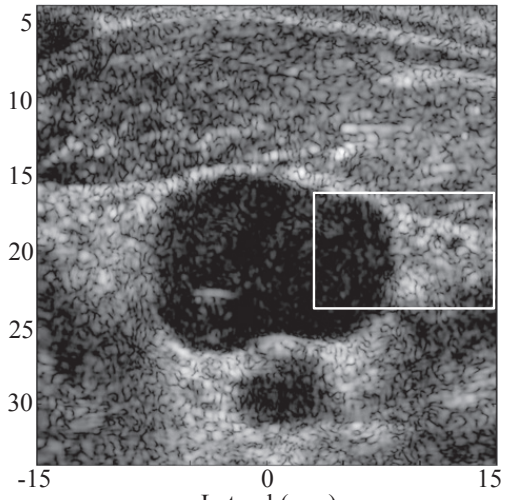

Lateral (mm)

(b)

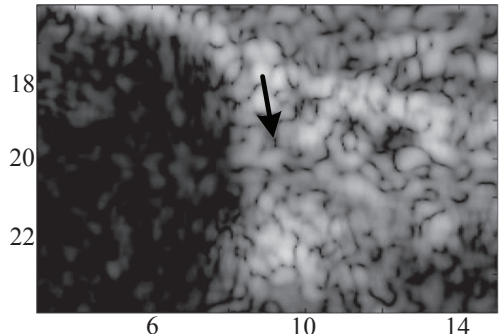

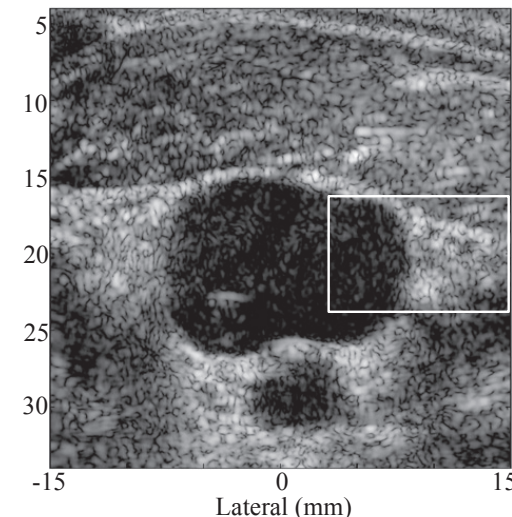

(c)

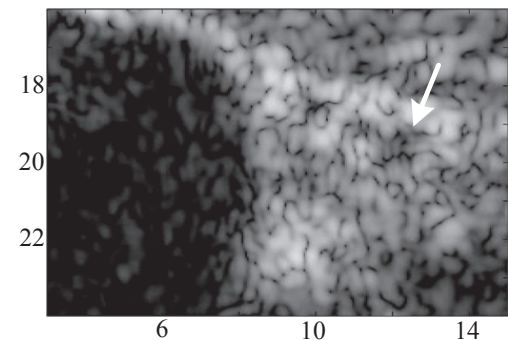

Fig. 8. (Top) The B-Mode image of the internal jugular vein (IJV) and common carotid artery (CCA) in transverse view. Both are surrounded by the thyroid gland (TG). The images are generated and displayed using (a) dynamic focusing, (b) unified PB beamforming, and (c) coherent PB beamforming. Labels in (a) are also relevant to the corresponding regions in images (b) and (c). Images in (b) and (c) are generated with data from 32 transmits. (Bottom) Magnified views of the regions enclosed by the white rectangles in the top images. All images are log-compressed and displayed with a dynamic range of $50 \mathrm{~dB}$.

$W_{1}$ and $W_{2}$, used to specify the apodization for the unified PB (see Fig. 1(c)), are enlarged to approximately four and eight times of the azimuthal resolution. The generated unified PB image with the matched apodization is displayed in Fig. 6(d). The speckle patterns look almost the same as those shown in Fig. 6(b), indicating no gain in spatial resolution. We also demonstrate the impact of the phase inversion used in the coherent $\mathrm{PB}$ by generating another coherent $\mathrm{PB}$ image without it. The result is shown in Fig. 6(e). In the new coherent PB image, there is a horizontal line across at the focal depth indicating the loss of coherence in the reconstruction.

For each image, we measure the speckle size and eSNR and summarized the results in Table II. In the near-field, the pressure field contains a quadratic phase factor [22] that causes fluctuations in the wavefront. As a result, the speckle granularity is not a valid indication of resolution and the correlation-based metric may not provide an accurate evaluation. We therefore measure the speckle sizes in the farfield and in the focal region, where the effects caused by the quadratic phase factor are much less. The eSNR is calculated on homogenous regions from $16 \mathrm{~mm}$ to $24 \mathrm{~mm}$, at the two sides of the lesion to avoid effects from strong reflectors. In the table, it shows that the unified PB image generated with the matched apodization to the coherent PB (unified PB with wider $W_{1,2}$ ) has lowest eSNR. This indicates the advantage of selecting focussed signals in the coherent PB approach. Furthermore, by using wider apodization in the unified PB, we lower the eSNR of the generated image for no gain in the spatial resolution.

To further illustrate the improvements of the coherent $\mathrm{PB}$, we demonstrate the beamformers on a second experimental dataset. Imaging data is acquired using the same transducer and system parameters but with the center frequency reduced to $3.5 \mathrm{MHz}$. The scan contains an anechoic lesion, 5-mm in diameter, positioned at $23.5 \mathrm{~mm}$. Images generated with different beamformers are shown in Figs. 7(a)-(d). They are respectively for: dynamic focusing, unified and coherent $\mathrm{PB}$, and the unified $\mathrm{PB}$ beamformer with apodization matched to the coherent PB. Fig. 7(c) shows that the speckle pattern is the finest at the focal depth in the coherent PB images. At this lower center frequency, the broader speckle sizes at the focal depth in the unified PB images are shown clearly in Figs. 7(b) and (d); especially in the magnified views. Similar metrics to those in Table II, but measured on these images, are calculated and summarized in Table III.

\section{E. In vivo study}

We acquired ultrasound imaging data from an ultrasound carotid experiment with a 53 year-old volunteer using the ULA-OP system. The experiment was conducted with appropriate ethical clearance and informed consent. The image generated with conventional dynamic focusing is shown in Fig. 8(a). It is a cross-sectional view of the internal jugular vein (IJV), the common carotid artery (CCA), and surrounding structures. These vessels carry the oxygen-rich blood cells up to the brain and down to the heart. In the image, the IJV and the CCA are displayed as anechoic regions, resulting in deeper structures appearing to have higher echogenicity (enhancement).

We applied the unified and coherent PB beamformers to the 
pre-beamformed data and show the results in Figs. 8(b) and (c). Both have much higher resolution than dynamic focussing, which is consistent with the results observed in our simulation and phantom studies. Improvements in the contrast can be seen clearly in the region of the CCA in the images generated by pixel-based algorithms. To show the difference between the unified and coherent PB beamformer, we have magnified a portion of each image including the IJV boundaries around $20 \mathrm{~mm}$ (enclosed by white rectangles in each of Figs. 8(a)(c)). On the magnified view of Fig. 8(b), there is blurring on the region at the focal depth from the unified PB (indicated with the black arrow), while the surrounding tissue is displayed with a finer speckle pattern in the corresponding region of the coherent PB image. We measure the coherence length of the speckle in these regions. The metric with the unified PB is around $0.58 \mathrm{~mm}$, while that measured with the coherent $\mathrm{PB}$ is approximately $0.43 \mathrm{~mm}$. In the figures, there are three small patches of reduced scattering in the coherent PB image that are less visible than those on the unified PB image (the biggest one is indicated with the white arrow). It is unclear whether these are small blood vessels or artefacts in the generated images. This, however, may indicate a loss of contrast which is in agreement with the higher sidelobes of the coherent $\mathrm{PB}$ approach, shown in the point-target simulation. The imaging results overall show that the improvements of the coherent $\mathrm{PB}$ over the unified $\mathrm{PB}$ in resolution are preserved in an in vivo context.

\section{Summary AND CONCLUSIONS}

In this paper, we have extended our pressure field analysis and propose the coherent PB beamformer as a new higher resolution approach to the computation of ultrasound B-scan images. Similar to the unified PB, the coherent PB is also designed to accumulate the data associated with the highest energy pulses. When neither pulse dominates the wave-shape, however, it picks data associated with both pulses and combines them with appropriate phase matching. The coherent PB algorithm has been demonstrated using simulations, experimental and in vivo data. It shows improvements in spatial resolution over the unified PB approach around the focal depth, and makes it optimal over the entire imaging region.

The coherent PB beamformer was developed in parallel with the minimum-variance-based beamformer in the companion paper [13]. They are based on similar frameworks of field pattern analysis. Compared to the minimum-variance algorithm, the coherent PB approach may have lower spatial and contrast resolution for scattering fields that comply with certain statistical restrictions. However, coherent PB is a faster, more robust method, and can work on any type of scatterer field. Like the unified PB, the coherent PB collects signals based on a time delay calculation. This limits the coherent PB to the use of one or two data points in each received waveform. Improvements offered by the beamformer, therefore, are limited to the lateral resolution. The axial resolution could be improved if some deconvolution could be incorporated into the beamformer to reduce the temporal correlation in the received waveforms. This motivates our future work.

\section{ACKNOWLEDGMENT}

We thank Dr. Laurence Berman of Addenbrooke's Hospital, Cambridge, UK for performing the in vivo scan and advising on its interpretation. We are also grateful to the anonymous reviewers for their insightful comments and helpful suggestions.

\section{REFERENCES}

[1] J. S. Hwang and T. K. Song, "A study of the display pixel-based focusing method in ultrasound imaging," in Ultrasonic Imaging, vol. 23 no. 1, pp. 1-18, 2001.

[2] Y. Lee, W. Y. Lee, C. E. Lim, J. H. Chang, T. K. Song, and Y. Yoo, "Compounded direct pixel beamforming for medical ultrasound imaging," in IEEE Trans Ultrason Ferroelec Freq Control, vol. 59, no. 3, pp. 573-582, 2012.

[3] J. A. Jensen, O. Holm, L. J. Jensen, H. Bendsen, S. Nikolov, B. G. Tomov, P. Munk, M. Hansen, K. Salomonsen, J. Hansen, K. Gormsen, M. H. Pedersen, and G. K. Ammelmark, "Ultrasound research scanner for real-time synthetic aperture data acquisition," in IEEE Trans Ultrason Ferroelec Freq Control, vol. 52, no. 5, pp. 881-891, 2005

[4] J. A. Jensen, S. I. Nikolov, K. L. Gammelmark, and M. H. Pedersen, "Synthetic aperture ultrasound imaging," in Ultrasonics, vol. 44, suppl., pp. e5-e15, 2006.

[5] R. E. Daigle, "Ultrasound imaging system with pixel oriented processing," U.S. Patent 2009/0112095 A1, May 19, 2009.

[6] M. H. Bae and M. K. Jeong, "A study of synthetic-aperture imaging with virtual source elements in B-mode ultrasound imaging systems," in IEEE Trans Ultrason Ferroelec Freq Control, vol. 47, no. 6, pp. 1510-1519, 2000

[7] C. Pass and H. Ermert, "A 100-MHz ultrasound imaging system for dermatologic and ophthalmologic diagnostics," in IEEE Trans Ultrason Ferroelec Freq Control, vol. 43, no. 4, pp. 545-551, 1996.

[8] C. Frazer and W. D. O'Brien Jr., "Synthetic aperture techniques with a virtual source element," in IEEE Trans Ultrason Ferroelec Freq Control, vol. 45, no. 1, pp. 196-206, 1998.

[9] R. Zemp and M. F. Insana, "Imaging with unfocused regions of focused ultrasound beams" in Journal of the Acoustical Society of America, vol. 121, no. 3, pp. 1491-1498, 2007.

[10] H. Andresen, S. Nikolov, and J. A. Jensen, "Precise time-of-flight calculation for 3-D synthetic aperture focusing," in IEEE Trans Ultrason Ferroelec Freq Control, vol. 56, no. 9, pp. 1880-1887, 2009.

[11] C. Kim, C. Yoon, J. H. Park, Y. Lee, W. H. Kim, J. M. Chang, B. I. Choi, T. K. Song, and Y. M. Yoo, "Evaluation of ultrasound synthetic aperture imaging using bidirectional pixel-based focusing: preliminary phantom and in vivo breast study," in IEEE Trans Biomedical Engineering, vol. 60, no. 10, pp. 2716-2724, 2013.

[12] N.Q. Nguyen and R.W. Prager, "High-resolution ultrasound imaging with unified pixel-based beamforming," IEEE Trans Med Imag, vol. 35, no. 1, pp. 98-108, 2016.

[13] N.Q. Nguyen and R.W. Prager, "Minimum variance approaches to ultrasound pixel-based beamforming," IEEE Trans Med Imag, vol. 36, no. 2, pp. 374-384, 2017.

[14] J. A. Jensen and N. B. Svendsen, "Calculation of pressure fields from arbitrarily shaped, apodized, and excited ultrasound transducers," IEEE Trans Ultrason Ferroelec Freq Control, vol. 39, no. 2, pp. 262-267, 1992.

[15] J. A. Jensen, "Field: A program for simulating ultrasound systems," Med Biol Eng Comp, vol. 34, Supplement 1, Part 1, pp. 351-353, 1996.

[16] P. Tortoli, L. Bassi, A. Dallai, F. Guidi, and S. Ricci, "ULA-OP: An advanced open platform for ultrasound research," in IEEE Trans Ultrason Ferroelec Freq Control, vol. 56 no. 10, pp. 2207-2216, 2009.

[17] R. S. C. Cobbold, Foundations of biomedical ultrasound. New York, NY: Oxford University Press, 2007.

[18] K. Mustafe, P-C. Li, and M. O'Donnell, "Synthetic aperture imaging for small scale systems," in IEEE Trans Ultrason Ferroelec Freq Control, vol. 42, no. 3, pp. 429-442, 1995.

[19] R.F. Wagner, S.W. Smith, J.M. Sandrik, and H. Lopez, "Statistics of speckle in ultrasound B-scans," IEEE Trans Son Ultrason, vol. 30, no. 3, pp. 156-163, 1983.

[20] S.W. Smith, R.F. Wagner, J.M. Sandrik, and H. Lopez, "Low contrast detectability and contrast/detail analysis in medical ultrasound," IEEE Trans Son Ultrason, vol. 30, no. 3, pp. 164-173, 1983. 
[21] S. Mehdizadeh, A. Austeng, T.F. Johansen, and S. Holm, "Eigenspace based minimum variance beamforming applied to ultrasound imaging of acoustically hard tissues," IEEE Trans Med Imag, vol. 31, no. 10, pp. 1912-1921, 2012.

[22] M.F. Insana, T. J. Hall, and L. T. Cook, "Backscatter coefficient estimation using array transducer," IEEE Trans Ultrason Ferroelec Freq Control, vol. 41, no. 5, pp. 714-723, 1994. 